\title{
Strict bladder filling and rectal emptying during prostate SBRT: Does it make a dosimetric or clinical difference?
}

\author{
David J. Byun', Daniel J. Gorovets², Lauren M. Jacobs², Laura Happersett ${ }^{3}$, Pengpeng Zhang ${ }^{3}$, Xin Pei ${ }^{4}$, \\ Sarah Burleson ${ }^{3}$, Zhigang Zhang ${ }^{4}$, Margie Hunt ${ }^{3}$, Sean McBride ${ }^{2}$, Marisa A. Kollmeier ${ }^{2}$ and Michael J. Zelefsky ${ }^{2^{*}}$ \\ Prior presentations: This abstract was presented at the 2016 American Society for Radiation Oncology \\ Meeting, September 25-28, 2016 in Boston, Massachusetts.
}

\begin{abstract}
Background: To evaluate inter-fractional variations in bladder and rectum during prostate stereotactic body radiation therapy (SBRT) and determine dosimetric and clinical consequences.

Methods: Eighty-five patients with 510 computed tomography (CT) images were analyzed. Median prescription dose was $40 \mathrm{~Gy}$ in 5 fractions. Patients were instructed to maintain a full bladder and empty rectum prior to simulation and each treatment. A single reviewer delineated organs at risk (OARs) on the simulation (Sim-CT) and Cone Beam CTs (CBCT) for analyses.

Results: Bladder and rectum volume reductions were observed throughout the course of SBRT, with largest mean reductions of $86.9 \mathrm{~mL}$ (19.0\%) for bladder and $6.4 \mathrm{~mL}$ (8.7\%) for rectum noted at fraction \#5 compared to Sim-CT $(P<0.01)$. Higher initial Sim-CT bladder volumes were predictive for greater reduction in absolute bladder volume during treatment $(\rho=-0.69 ; P<0.01)$. Over the course of SBRT, there was a small but significant increase in bladder mean dose $(+4.5 \pm 12.8 \% ; P<0.01)$ but no significant change in the D2cc $(+0.8 \pm 4.0 \% ; P=0.28)$. The mean bladder trigone displacement was in the anterior direction $(+4.02 \pm 6.59 \mathrm{~mm})$ with a corresponding decrease in mean trigone dose $(-3.6 \pm 9.6 \% ; P<0.01)$ and D2cc $(-6.2 \pm 15.6 \% ; P<0.01)$. There was a small but significant increase in mean rectal dose $(+7.0 \pm 12.9 \%, P<0.01)$ but a decrease in rectal D2cc $(-2.2 \pm 10.1 \% ; P=0.04)$. No significant correlations were found between relative bladder volume changes, bladder trigone displacements, or rectum volume changes with rates of genitourinary or rectal toxicities.

Conclusions: Despite smaller than expected bladder and rectal volumes at the time of treatment compared to the planning scans, dosimetric impact was minimal and not predictive of detrimental clinical outcomes. These results cast doubt on the need for excessively strict bladder filling and rectal emptying protocols in the context of image guided prostate SBRT and prospective studies are needed to determine its necessity.
\end{abstract}

Keywords: SBRT, Prostate cancer, Bladder volume, Rectum volume, Interfractional organ displacement

*Correspondence: zelefskm@mskcc.org

2 Department of Radiation Oncology, Memorial Sloan Kettering Cancer Center, 1275 York Ave, New York, NY 10065, USA

Full list of author information is available at the end of the article

\section{Background}

Stereotactic body radiation therapy (SBRT) is gaining momentum for its clinical application of the treatment of localized prostate cancer. The use of SBRT is becoming a original author(s) and the source, provide a link to the Creative Commons licence, and indicate if changes were made. The images or other third party material in this article are included in the article's Creative Commons licence, unless indicated otherwise in a credit line to the material. If material is not included in the article's Creative Commons licence and your intended use is not permitted by statutory regulation or exceeds the permitted use, you will need to obtain permission directly from the copyright holder. To view a copy of this licence, visit http://creativecommons.org/licenses/by/4.0/. The Creative Commons Public Domain Dedication waiver (http://creativeco mmons.org/publicdomain/zero/1.0/) applies to the data made available in this article, unless otherwise stated in a credit line to the data. 
viable alternative with preliminary clinical trials and single institutions reporting equal or superior biochemical recurrence rates to the traditional forms of external beam radiation therapy (EBRT) and radical prostatectomy [111]. However, the accuracy of radiation is more critical for SBRT in comparison to conventional EBRT given its higher dose per fraction, minimal margin for error in target volume localization, and sensitivity of late responding normal tissue to hypofractionation [12]. Thus far, reports of toxicity outcomes after SBRT have been encouraging. Comparable rates of genitourinary and bowel toxicities to IMRT have been reported in preliminary trials and multi-institutional experiences $[1,4-6,13]$. Recently, a Phase III non-inferiority clinical trial comparing conventional prostate EBRT to a seven-fraction prostate SBRT regimen (4270 cGy) revealed a comparable acute end-oftreatment urinary and bowel toxicity outcomes, as well as at 5-year follow-up [7].

We hypothesize that variations in bladder and rectum filling during the course of prostate SBRT could result in unexpected doses being delivered to critical normal tissues and possibly affect the rate of treatment-related toxicity. To our knowledge, an evaluation of anatomic variations in the bladder and rectal filling in an SBRT prostate cancer cohort has yet to be conducted. To this end, we sought to determine the amount of interfractional pelvic organ volume changes and displacement during prostate SBRT, as well as the potential dosimetric and clinical impact on these organs at risk (OARs) in a cohort of patients who were placed on a strict pre-treatment bladder filling and rectal emptying pre-treatment regimen.

\section{Methods \\ Patient population, simulation procedures and target volumes}

Following Institutional Review Board approval, data were obtained from medical records of 85 consecutive patients treated with 5-fraction SBRT to the prostate from September 2014 to August 2015 at our institution. Each patient underwent a $2-3 \mathrm{~mm}$ slice thickness simulation computed tomography (Sim-CT) in the supine position with thermoplast immobilization (Aquaplast; Qfix, Avondale, PA). To maintain an adequately filled bladder for simulation and treatment, patients were instructed to drink 2 cups of water $1 \mathrm{~h}$ prior to each session. Strict rectal protocol of fiber supplementation, simeticone, and rectal enemas $3 \mathrm{~h}$ prior to simulation and each treatment was advised. During the course of this study, rectal spacers were not used in this patient cohort. Based on the simulation $\mathrm{CT}$ and fusion of available pre-treatment prostate MRI, clinical target volume (CTV) was defined as prostate gland plus proximal $\geq 1 \mathrm{~cm}$ of the seminal vesicles. The planning target volume (PTV) was defined as CTV expansion of $5 \mathrm{~mm}$ throughout except for $3 \mathrm{~mm}$ posterior.

\section{Organ displacement management and delineation}

To adjust for any inter-fractional clinical target volume (CTV) shift, kV orthogonal radiographs and Cone Beam CT (CBCT) images were acquired prior to each treatment to realign intraprostatic fiducial markers to the planned CTV position that had been determined at the Sim-CT. Treating physicians visually assessed the adequacy of bladder filling and rectal volumes on the CBCTs compared to initial Sim-CT prior to treatment delivery.

Using the Eclipse ${ }^{\circledR}$ Treatment Planning System (Varian Medical Systems; Palo Alto, CA), CBCTs were registered and blended with the Sim-CT for each patient. A single reviewer determined anatomical delineations of the CTV (prostate and seminal vesicles), bladder wall, bladder trigone, and rectum on the baseline Sim-CT, as well as the 5 blended CBCTs, for each patient $(n=510$ total images). For each scan, the rectum was contoured from the rectosigmoid flexure superiorly to the ischial tuberosity inferiorly. Consistency of rectal length was maintained through all 6 sets of CT images for each patient. Bladder trigone was defined as the triangular posteroinferior region of the bladder wall extending from the ureteral orifices superiorly to the internal urethral sphincter inferiorly.

\section{Volume, position, and dosimetric evaluation}

Target and OAR volumes were obtained from the treatment planning system. Interfractional organ displacements were quantified by calculating the organ center of mass (COM) differences on the individual pre-treatment CBCTs aligned to CTV and intraprostatic fiducial markers in relation to the baseline Sim-CTs. To account for the reproducibility of the pelvic organ delineation by the reviewer, 10 randomly selected CBCTs were re-contoured at the end of data collection. Intraclass correlation coefficient (ICC) was used to evaluate the level of reproducibility in the organ center of mass displacements and organ volumes, where a coefficient of 1 implies perfect reproducibility and 0 implies no consistency in delineation by the single reviewer [14].

CBCTs were fused to Sim-CT and fractional doses for the newly delineated $\mathrm{CBCT}$ structures were recalculated based on the original treatment plan. To account for any variation in dose prescriptions and OAR dimensional variations at the level of the CTV, fractional mean OAR doses and fractional maximum doses to $2 \mathrm{cc}$ of OAR (D2cc) were represented as a percentage of the initial dose at simulation. 


\section{Statistical analysis}

A two-tailed Wilcoxon signed-rank test was used to evaluate differences between volume, displacement, mean doses, and D2cc of pelvic organs at Sim-CT compared to subsequent fractionations. Absolute inter-fraction volume change was defined as the volume at fraction \#5 subtracted from the volume at Sim-CT (denoted $\left.V_{5-S i m}\right)$. Simple regression analysis (denoted $\mathrm{r}^{2}$ ) was used as a proxy to represent the degree of variance of intra-patient volume change through the course of treatment. Spearman's rank order correlation ( $\rho$ ) was used to assess if pretreatment International Prostate Symptom Score (IPSS) was predictive of bladder volume changes, and whether bladder and rectum volumes at Sim-CT was predictive of volume change, organ displacement, or dose variation.

A two-sided Wilcoxon rank sum test was implemented to evaluate whether relative bladder volume, rectum volume, and bladder trigone displacement variations were predictive of acute ( $<30$ days from completion of treatment) and late ( $\geq 30$ days from completion of treatment) genitourinary (GU) or gastrointestinal (GI) toxicities. GU/GI toxicities were defined based on the Common Terminology Criteria for Adverse Events version 5.0 and categorized as a clinically significant toxicity event if grade 2 or higher.

The significance level at which a null hypothesis would be rejected was defined as $P<0.05$. Statistical analyses were conducted using SPSS $^{\circledR}$ software Version 23.0 (IBM Corp.; Armonk, NY).

\section{Results}

\section{Patient characteristics}

Of the 85 total patients, 61 received prescription doses of $40 \mathrm{~Gy}$ in 5 fractions, 10 received prescription doses of 37.5 Gy in 5 fractions, and 14 received combination therapy of low dose rate brachytherapy in addition to 5 -fraction SBRT to prescription doses of $25 \mathrm{~Gy}$. The mean age was 69.7 years old (range 51-85 years), with tumor stages ranging from $\mathrm{T}_{1-3} \mathrm{~N}_{0} \mathrm{M}_{0}(\mathrm{n}=79)$ to $\mathrm{T}_{1-4} \mathrm{~N}_{0-1} \mathrm{M}_{0-1}(\mathrm{n}=6)$. The median follow-up was 2.85 years.

\section{Reproducibility of organ delineation}

Comparisons of the initial pelvic organ delineation in 10 randomly chosen $\mathrm{CBC}$ Ts and the repeat delineations by a single reviewer showed excellent reproducibility in COM displacement as well as organ volume (ICC coefficient range 0.93-1.00).

\section{Bladder and rectum volume}

The mean bladder volume at the time of the baseline Sim-CT was $291.2 \pm 157.3 \mathrm{~mL}$. Bladder volumes in the subsequent CBCTs were as follows: $263.4 \pm 147.1 \mathrm{~mL}$ at fraction \#1, 236.6 $\pm 145.0 \mathrm{~mL}$ at \#2, $234.3 \pm 128.1 \mathrm{~mL}$ at $\# 3,220.0 \pm 127.6 \mathrm{~mL}$ at $\# 4$, and $204.3 \pm 106.9 \mathrm{~mL}$ at \#5 (Fig. 1). The average bladder volume reduction per patient from Sim-CT to fraction \#5 ( $\mathrm{V}_{5-\mathrm{Sim}}$ Bladder) significantly decreased by $86.9 \mathrm{~mL}$ or $19.0 \%$ (Wilcoxon signed-rank test: $P<0.01)$. In 10 patients, exact bladder volume could not be delineated due to limitations in field of view in CBCTs compared to full pelvic simulation CTs. After accounting for these limitations and excluding patients with sub-optimal CBCTs, $\mathrm{V}_{5 \text {-Sim }}$ Bladder significantly decreased by $90.2 \mathrm{~mL}$ or $20.6 \%$ (Wilcoxon signedrank test: $P<0.01)$. Sim-CT bladder volume was inversely correlated with $\mathrm{V}_{5 \text {-Sim }}$ Bladder $(\rho=-0.69 ; P<0.01)$; thus, larger bladder volume at simulation was predictive of larger bladder volume loss toward fraction \#5 (Fig. 2a). However, pre-treatment IPSS score, a metric for urinary dysfunction, was not predictive of relative bladder volume reduction through treatment $(\rho=-0.07 ; P=0.5)$. Sim-CT bladder volume was not a significant predictor of variance in intra-patient bladder volume change $\left(\mathrm{r}^{2}\right)$ (Spearman's correlation $=0.18 ; P=0.09$ ) (Fig. 2b).

Baseline Sim-CT rectum volume was $56.1 \pm 19.6 \mathrm{~mL}$, with subsequent rectum volumes measuring $54.4 \pm 23.6 \mathrm{~mL}$ at fraction \#1, $54.1 \pm 21.7 \mathrm{~mL}$ at $\# 2,52.7 \pm 21.5 \mathrm{~mL}$ at $\# 3,53.1 \pm 24.2 \mathrm{~mL}$ at \#4, and $49.6 \pm 20.7 \mathrm{~mL}$ at $\# 5$ (Fig. 3). Rectum volume significantly decreased by $6.4 \mathrm{~mL}$ or $8.7 \%$ from Sim-CT to fraction \#5 $\left(\mathrm{V}_{5 \text {-Sim }}\right.$ Rectum) (Wilcoxon signed-rank test: $\left.P<0.01\right)$. A weak but significant association between the Sim$\mathrm{CT}$ rectum volume and the $\mathrm{V}_{5 \text {-Sim }}$ Rectum was observed

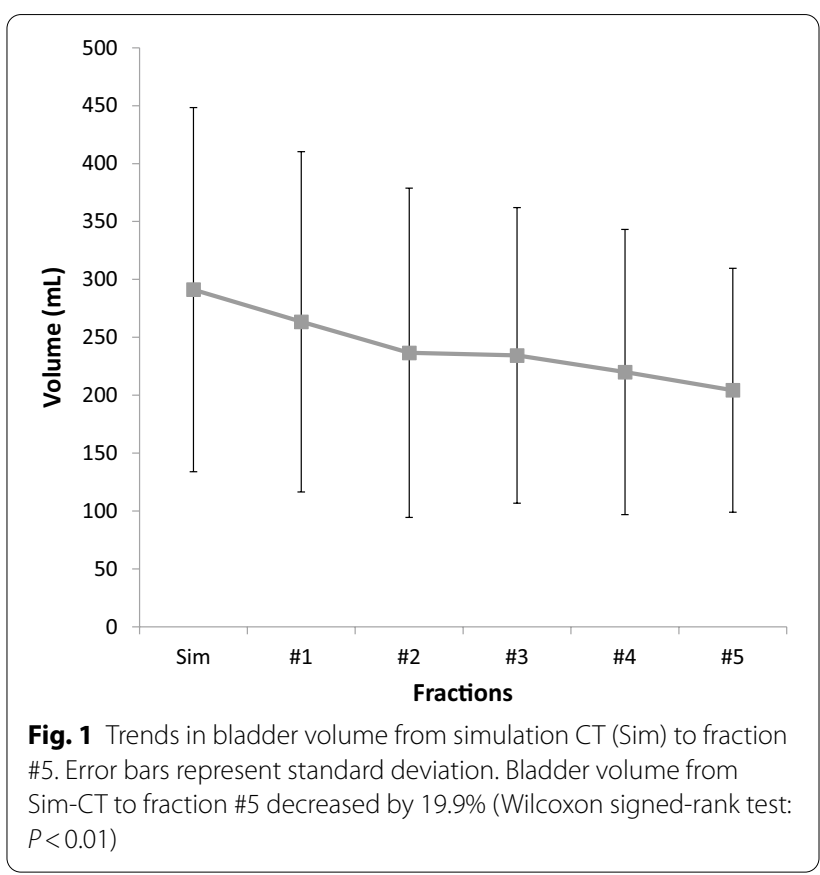




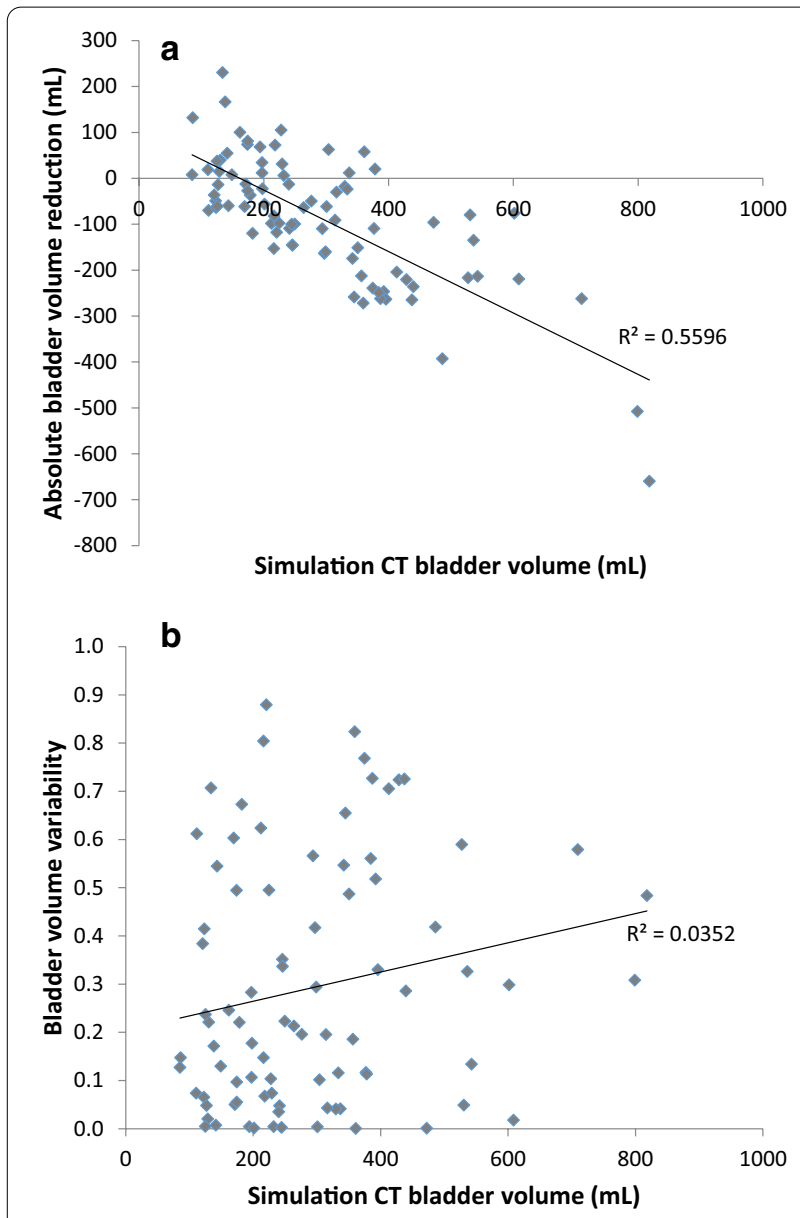

Fig. 2 a Sim CT volume in relation to absolute volume reduction $\left(V_{5-\text { sim }}\right)$. The goodness of fit of the linear regression model is represented by $R^{2}$ (Spearman's correlation $=-0.69 ; P<0.001$ ). b Sim CT volume in relation to bladder volume variability $\left(r^{2}\right)$ in individual patients through 5 fractions. The goodness of fit of the linear regression model is represented by $R^{2}$ (Spearman's correlation $=0.18$; $P=0.09)$

(Spearman's correlation $=-0.51 ; \quad P<0.01)$; however, Sim-CT rectum volume was not a predictor of rectum $r^{2}$ (Spearman's correlation $=0.08 ; P=0.48$ (Fig. 4a, b).

\section{Pelvic organ displacement}

Patients were positioned for treatment based on intraprostatic fiducial marker matching, therefore the prostate CTV COM had minimal inter-fractional displacements and typically remained within our PTV margins (lateral: $-0.11 \pm 0.82 \mathrm{~mm}$; anterior/posterior: $+0.64 \pm 1.75 \mathrm{~mm}$; superior/inferior: $+0.07 \pm 1.37 \mathrm{~mm}$ ). However, OAR displacements were more pronounced (Table 1). Increased inferior bladder (all fractions: $-2.82 \pm 8.98 \mathrm{~mm}$, fraction 5: $-3.40 \pm 8.84 \mathrm{~mm}$ ), anterior bladder trigone (all fractions: $+2.64 \pm 6.25 \mathrm{~mm}$; fraction $5:+4.02 \pm 6.59 \mathrm{~mm})$,

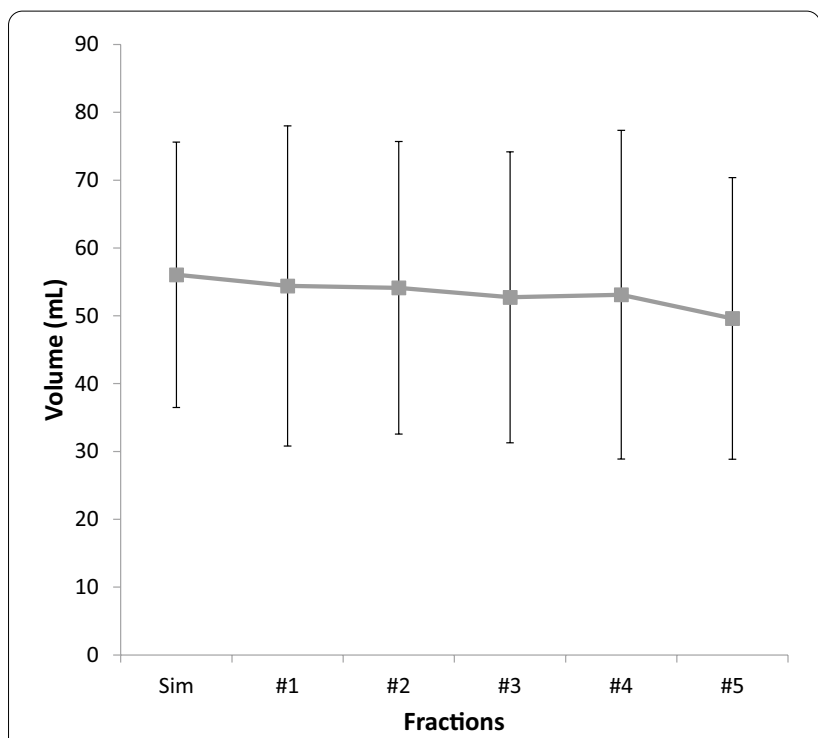

Fig. 3 Trends in rectum volume from simulation CT (Sim) to fraction \#5. Error bars represent standard deviation. Rectum volume from Sim-CT to fraction \#5 decreased by $8.7 \%$ (Wilcoxon signed-rank test: $P<0.01)$

and superior rectum (all fractions: $+3.19 \pm 5.84 \mathrm{~mm}$; fraction $5:+2.38 \pm 5.74 \mathrm{~mm}$ ) displacements were observed during all fractions compared to the initial COM at Sim-CT. Further, the percentages of inter-fractional displacements surpassing $3 \mathrm{~mm}$ were considerable in the inferior bladder (all fractions: $75 \%>3 \mathrm{~mm}$; fraction 5: $82 \%>3 \mathrm{~mm}$ ), anterior bladder trigone (all fractions: $53 \%>3 \mathrm{~mm}$; fraction $5: 52 \%>3 \mathrm{~mm}$ ), and superior rectum displacements (all fractions: $62 \%>3 \mathrm{~mm}$; fraction 5: $65 \%>3 \mathrm{~mm})$.

\section{Pelvic organ dosimetry}

Over the course of SBRT, there was a significant but small increase in bladder mean dose (all fractions: $+3.7 \pm 13.6 \%$; fraction $5:+4.5 \pm 12.8 \%$; $P<0.01$ ), a decrease in bladder trigone mean dose (all fractions: $-2.1 \pm 11.0 \%$; fraction $5:-3.6 \pm 9.6 \%, P<0.01)$, and an increase in rectum mean dose (all fractions: $+8.4 \pm 14.3 \%$; fraction $5:+7.0 \pm 12.9 \%, P<0.01)$ compared to baseline Sim-CT (Table 2). Evaluating for maximum dose exposure to OARs, there was no significant change in bladder D2cc dose (all fractions: $+0.4 \pm 2.7 \%$; fraction $5:+0.8 \pm 4.0 \% ; P=0.28)$, a small but significant decrease in bladder trigone D2cc dose (all fractions: $-3.0 \pm 23.9 \%$; fraction $5:-6.2 \pm 15.6 \% ; P<0.01)$, and a small but significant decrease in rectum D2cc dose (all fractions: $-1.0 \pm 10.0 \%$; fraction $5:-2.2 \pm 10.1 \% ; P=0.04$ ) from simulation D2cc. 


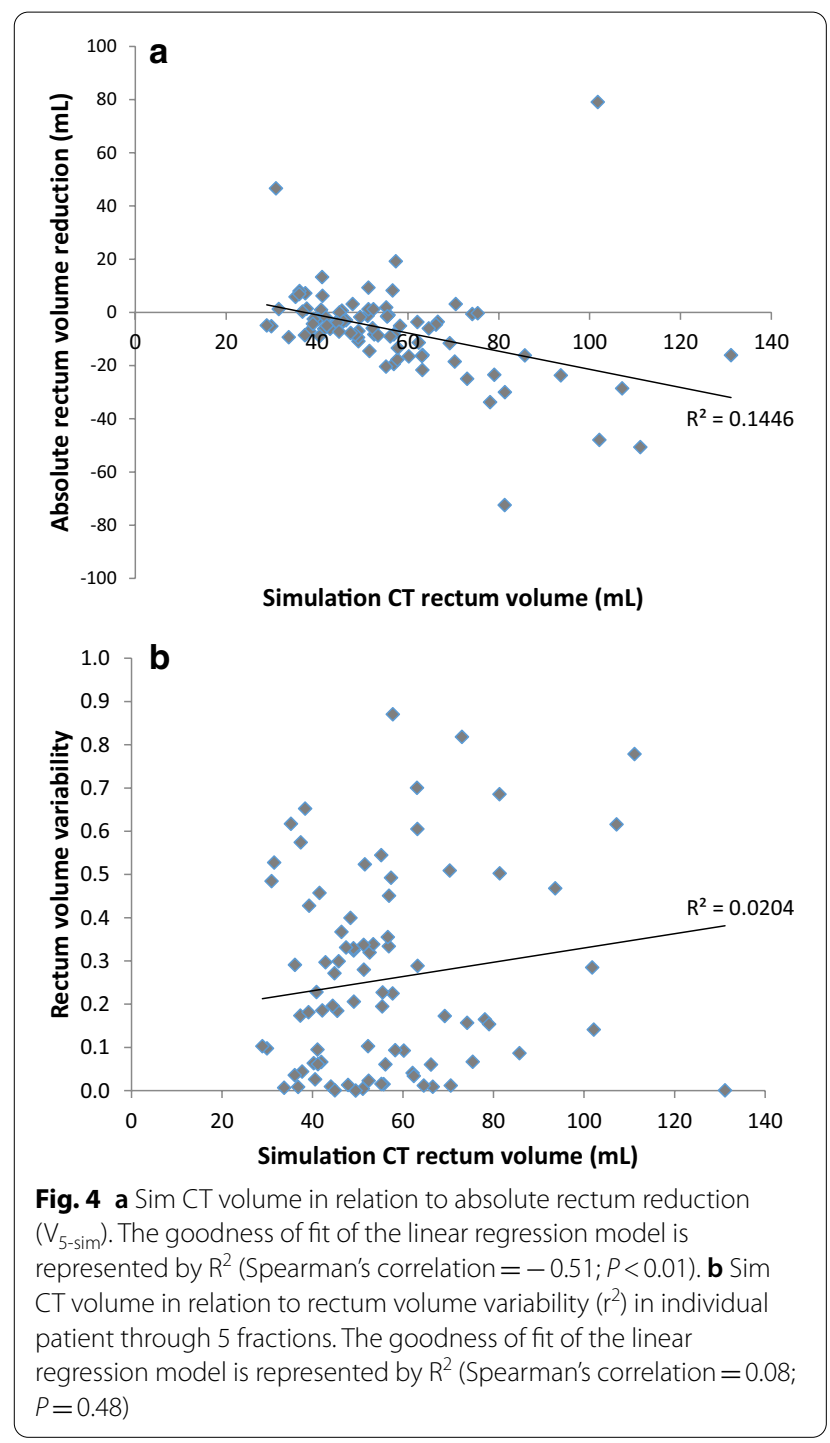

Volume as a predictor of displacement and dose

A significant correlation was found between $\mathrm{V}_{5 \text {-Sim }}$ Bladder (bladder volume reduction over the course of treatment) and anterior displacement of the bladder trigone $(\rho=-0.50 ; P<0.001)$. Anterior bladder trigone displacement was also significantly associated with SimCT bladder volume $(\rho=0.30 ; P=0.005)$ and $r^{2}(\rho=0.31$; $P=0.004)$. Despite these findings, bladder trigone dosimetry was not significantly associated with $\mathrm{V}_{5 \text {-Sim }}$ Bladder $(P=0.12), V_{\text {Sim-CT }}$ Bladder $(P=0.35)$, or $\mathrm{r}^{2} \quad(P=0.94)$. $\mathrm{V}_{5 \text {-Sim }}$ Rectum (rectal volume reduction over the course of treatment) was associated with superior rectal displacement $(\rho=0.27 ; P=0.01)$ and mean rectal dose change $(\rho=0.27 ; P=0.01)$. Rectal volume variation and volume at Sim-CT were not significantly associated with rectal displacement $(P=0.89 ; P=0.92$, respectively) or dosimetry $(P=0.61 ; P=0.98$, respectively).

\section{Organ volume and position as predictors} of treatment-related sequalae

In 71 patient who underwent single modality SBRT, clinically significant acute GU toxicity (grade 2) were observed in $12.7 \%$, late grade $2 \mathrm{GU}$ toxicity in $35.2 \%$ of patients (Table 3 ). No clinically significant acute GI toxicity events were seen and only $4.3 \%$ of patients presented with late grade 2 GI toxicity. No significant correlations were found between relative bladder volume changes, rectum volume changes, or anterior bladder trigone displacements with rate of GU/GI toxicities in 71 patients who underwent single modality SBRT alone. Relative bladder volume changes from simulation to fraction 5 were not predictive of acute GU toxicity events $(P=0.47)$ or late $\mathrm{GU}$ toxicity events $(P=0.28)$. Anterior bladder trigone displacement was also not significantly associated with an increase in acute GU toxicity $(P=0.27)$ or late $\mathrm{GU}$ toxicity $(P=0.39)$. Due to the lack of acute GI toxicity events $(n=0)$ and insufficient late GI toxicity events $(n=3 ; 4.3 \%)$, data were not sufficient for statistical analysis. Patients who received combination SBRT and brachytherapy $(n=14)$ were excluded from toxicity analysis to account for the possible confounding effect of toxicities related to low dose rate seed placement.

\section{Discussion}

Optimal bladder and rectal filling for external beam radiation therapy for prostate cancer is a key topic of interest [15-17]. Full bladder protocols have the potential to reduce OAR dose exposure via displacement of the small bowel and bladder away from the target volume, with the caveat of increased volume variability [18-20]. Similarly, smaller rectal volumes can reduce dose exposure, but are more difficult to reproduce. Although we could infer from the findings of conventional EBRT cohort evaluation, to our knowledge, there has been no prior evaluation or report of inter-fraction OAR displacement and the dosimetric implications in patients undergoing image-guided prostate SBRT.

Despite instructions to maintain a consistently full bladder at simulation and during treatment, we observed volume variability and a systematic decline in bladder volume through the course of therapy with the largest reduction at the 5 th fraction. We hypothesize that this could be related to either radiation-related bladder capacity changes due to acute inflammation, increased efficiency of patient setup, and/or diminished adherence to the filling protocol as the treatment course progressed. Within the cohort analyzed, pre-treatment urinary dysfunction reflected by IPSS score was not associated with decline in bladder volume during treatment. 
Table 1 Displacement of pelvic organ center of mass compared to simulation CT (in $\mathrm{mm}$ )

\begin{tabular}{|c|c|c|c|c|}
\hline & \multicolumn{2}{|l|}{ All fractions } & \multicolumn{2}{|l|}{ Fraction 5} \\
\hline & Mean \pm SD & $>3 \mathrm{~mm}(\%)^{\mathrm{a}}$ & Mean \pm SD & $>3 \mathrm{~mm}(\%)^{\mathrm{a}}$ \\
\hline \multicolumn{5}{|l|}{$C T V^{b}$} \\
\hline Lateral & $-0.11 \pm 0.82$ & 1 & $-0.07 \pm 0.87$ & 1 \\
\hline Anterior/posterior & $0.64 \pm 1.75$ & 10 & $0.66 \pm 1.73$ & 12 \\
\hline Superior/inferior & $0.07 \pm 1.37$ & 3 & $0.37 \pm 1.44$ & 6 \\
\hline \multicolumn{5}{|l|}{ Bladder } \\
\hline Lateral & $-0.03 \pm 2.84$ & 26 & $0.02 \pm 2.91$ & 27 \\
\hline Anterior/posterior & $-0.70 \pm 6.45$ & 60 & $-0.86 \pm 6.24$ & 62 \\
\hline Superior/inferior & $-2.82 \pm 8.98$ & 75 & $-3.40 \pm 8.84$ & 82 \\
\hline \multicolumn{5}{|l|}{ Bladder Trigone } \\
\hline Lateral & $-0.19 \pm 1.64$ & 8 & $-0.14 \pm 1.90$ & 12 \\
\hline Anterior/posterior & $2.64 \pm 6.25$ & 53 & $4.02 \pm 6.59$ & 52 \\
\hline Superior/inferior & $-0.50 \pm 3.43$ & 8 & $-0.55 \pm 3.29$ & 6 \\
\hline \multicolumn{5}{|l|}{ Rectum } \\
\hline Lateral & $-0.13 \pm 1.86$ & 9 & $-0.12 \pm 1.87$ & 8 \\
\hline Anterior/posterior & $-0.21 \pm 3.28$ & 26 & $0.05 \pm 3.14$ & 24 \\
\hline Superior/inferior & $3.19 \pm 5.84$ & 62 & $2.38 \pm 5.74$ & 65 \\
\hline
\end{tabular}

Positive values denote superior, anterior, or leftward displacement of organ center of mass

a $>3 \mathrm{~mm}$ refers to percentage of $>3 \mathrm{~mm}$ displacements relative to center of mass at simulation CT

b CTV denotes prostate and seminal vesicles

Table 2 Trends in mean dose from simulation CT to fraction \#5

\begin{tabular}{lllll}
\hline & $\begin{array}{l}\text { Prostate } \\
\text { Mean } \pm \text { SD }\end{array}$ & $\begin{array}{l}\text { Bladder } \\
\text { Mean } \pm \text { SD }\end{array}$ & $\begin{array}{l}\text { Bladder Trigone } \\
\text { Mean } \pm \text { SD }\end{array}$ & $\begin{array}{l}\text { Rectum } \\
\text { Mean } \pm \text { SD }\end{array}$ \\
\hline Simulation & $102.80 \% \pm 1.00 \%$ & $42.10 \% \pm 18.30 \%$ & $93.50 \% \pm 9.20 \%$ & $43.90 \% \pm 6.40 \%$ \\
Fraction 1 & $102.50 \% \pm 1.00 \%$ & $44.50 \% \pm 18.80 \%$ & $92.50 \% \pm 9.90 \%$ & $47.90 \% \pm 8.70 \%$ \\
Fraction 2 & $103.40 \% \pm 8.10 \%$ & $46.80 \% \pm 20.80 \%$ & $92.10 \% \pm 9.60 \%$ & $47.50 \% \pm 7.70 \%$ \\
Fraction 3 & $102.50 \% \pm 1.00 \%$ & $44.80 \% \pm 19.80 \%$ & $90.90 \% \pm 10.80 \%$ & $47.20 \% \pm 8.30 \%$ \\
Fraction 4 & $102.50 \% \pm 1.00 \%$ & $46.40 \% \pm 20.50 \%$ & $90.20 \% \pm 10.10 \%$ & $47.10 \% \pm 7.30 \%$ \\
Fraction 5 & $102.10 \% \pm 3.20 \%$ & $46.70 \% \pm 19.90 \%$ & $89.80 \% \pm 10.50 \%$ & $46.60 \% \pm 7.10 \%$ \\
$\Delta$ dose (\%) & $-0.60 \% \pm 2.90 \%$ & $4.50 \% \pm 12.80 \%$ & $-3.60 \% \pm 9.60 \%$ & $7.00 \% \pm 12.90 \%$ \\
$\Delta$ dose (Gy) & $-0.2 \pm 1.1$ & $1.8 \pm 4.8$ & $-1.5 \pm 3.3$ & $0.96 \pm 1.87$ \\
\hline
\end{tabular}

$\triangle$ Dose denotes percentage or absolute mean dose change from simulation $\mathrm{CT}$ to fraction \#5. Fraction \#1-5 mean doses were significantly different from simulation dose in all OARs (all $P<0.01$ )

Table 3 Acute and late genitourinary and gastrointestinal toxicity events in single modality prostate SBRT cases $(\mathbf{N}=\mathbf{7 1})$

\begin{tabular}{llllll}
\hline & \multicolumn{2}{l}{ Genitourinary toxicities } & & \multicolumn{2}{c}{ Gastrointestinal toxicities } \\
\cline { 2 - 3 } & $\begin{array}{l}\text { <Grade 2 } \\
(\%)\end{array}$ & Grade 2 (\%) & & $\begin{array}{l}<\text { Grade 2 } \\
\text { (\%) }\end{array}$ & Grade 2 (\%) \\
\hline $\begin{array}{c}\text { Acute } \\
(<30 \text { days })\end{array}$ & $12(16.9)$ & $9(12.7)$ & & $2(2.8)$ & $0(0.0)$ \\
$\begin{array}{c}\text { Late } \\
(\geq 30 \text { days })\end{array}$ & $16(22.5)$ & $25(35.2)$ & & $11(15.5)$ & $3(4.3)$ \\
\hline
\end{tabular}

Several past evaluations of patients treated with conventionally fractionated EBRT with full bladder protocols have reported similar results [15, 21, 22]. For instance, a prospective study by Nakamura et al. [17] showed a significant decline of $38 \%$ in bladder volume from fraction $\# 1$ to \#30. To develop a feedback mechanism for consistent inter-fraction bladder volumes, Stam et al. [22] used daily bladder scans to provide patients with verbal feedback on fluid consumption to improve bladder filling. While there was a moderate improvement in volume 
reduction in the bladder scan group (19\% decline) compared to the control group (31\%), the authors concluded that the difference was not sufficient to deem their technique clinically applicable. In a series of 41 patients undergoing conventionally fractionated EBRT, O'Doherty et al. [23] found that using written instructions resulted in more consistent bladder filling throughout treatment, with an association between patient's subjective bladder fullness and actual bladder volume. Thus, written instructions may help patients maintain a moderately consistent bladder volume for prostate SBRT as well.

Our results, however, call into question the need for such strict bladder filling instructions when using modern radiotherapy techniques that employ more accurate MRI-based target delineation and smaller PTVs facilitated by advanced inter- and intra-fraction image guidance. While significant, it is uncertain if our observation of increased mean dose exposure to the bladder with a decline in bladder filling would ultimately translate to clinically significant GU sequelae in a prospective setting. Our retrospective assessment of the available acute and late GI and GU toxicities data certainly did not reveal a clear correlation to bladder volume and displacement variations.

To date, there have been relatively few defined dosevolume relationships for prostate SBRT that have predicted greater significant GU toxicity. Repka et al. [24] found bladder wall D15.5\% >32.6 Gy to be significantly associated with acute urinary toxicity. Therefore, the volume of the bladder receiving high doses might be more relevant than the mean dose. In the current study, bladder D2cc did not change significantly over the course of treatment, suggesting that the area of bladder exposed to high doses was similar to what we expected based on the DVHs generated during treatment planning. Additionally, anatomical sub-sites of the bladder or urethra (i.e., bladder trigone/neck or membranous urethra) could be relatively more important to avoid with high doses of radiation [25, 26]. Analysis of the bladder trigone was of specific interest given our previously published analyses of patients treated with conventionally fractionated EBRT and low-dose rate brachytherapy, which revealed a strong association between urinary toxicity and exposure to the bladder neck region [25, 26]. Overall bladder volume reduction over the course of SBRT observed in the current study was accompanied by a displacement of the trigone anteriorly and away from areas of highest dose exposure. This phenomenon may be explained by collapsing of the overextended bladder walls in the axial plane with a reduction in urine volume between fractions, as illustrated by the inferior shift in the center of mass of the bladder from simulation to the end of treatment. Anterior displacement of the bladder trigone may therefore represent a surrogate for changes in the surface area of the posterior bladder wall. While previous studies regarding bladder filling emphasized the importance of reproducibility of a full bladder to reduce urinary toxicity, the bladder volume reduction observed here and increased mean bladder dose may minimally contribute to toxicity if the D2cc remains unchanged and the trigone is simultaneously spared potentially deleterious dose exposure [27, 28]. Clearly further exploration of the optimal bladder filling for simulation and treatment in the setting of a highly precise treatment, such as prostate SBRT, is needed.

Although not as substantial as bladder volume reductions, rectal volume also showed a decreasing trend over the course of the 5 fraction SBRT, with a significant but small increase in mean dose exposure to the rectal wall but decrease in D2cc. These findings are corroborated by studies in conventionally fractionated EBRT involving full bladder protocols, in which rectal volume reductions were observed through the course of therapy $[16,19,29]$. It is also possible that the strict adherence to and cumulative effect of daily rectal emptying instructions involving fiber supplementation, simethicone, and enemas may partially explain the decreasing rectal volume over the treatment course. In addition, acute rectal symptoms with progression of treatment, namely tenesmus, may further propagate the rectal volume reduction observed [30]. While the literature concerning rectal displacement in SBRT patients is limited, a recent dosimetric study suggested that rectum volume receiving $75 \%$ of the prescribed dose can increase significantly during SBRT due to interfraction organ displacement [31]. As in bladder dose volume studies, prior studies of rectal displacement in relation to dose exposure involved conventionally fractionated EBRT employing more generous PTV margins and substantially greater number of fractions over several weeks and therefore may not be applicable to more precise ultra-hypofractionated treatments, especially considering the increased use of rectal spacers to mitigate rectal dose. Furthermore, while there are specific dose constraint guidelines for the rectum in conventional EBRT and brachytherapy, prospectively validated dose constraint guidelines for ultra-hypofractionated SBRT have yet to be established [32, 33].

Based on an evaluation of rectal tolerance in a Phase I/ II trial, Kim et al. [34] suggested that limiting 35\% rectal circumference exposure to less than 39 Gy over 5 fractions may reduce the risk for delayed rectal toxicity. Therefore, while studies have reported acceptable levels of rectal toxicity with prostate SBRT and our current assessment yielded nonexistent-to-exceedingly low rates of GI toxicities, ongoing prospective trials with longterm follow up must be evaluated before we can fully 
understand the dose-volume relationships and the potential impact of interfractional organ displacement $[1,5,13$, 35-38].

The key strengths of this study include its use of an objectively verified and consistent process of organ delineation, as well as its analysis of a large number of CT images. A limitation of this study was the inferior image quality produced by the CBCTs, which might have resulted in errors in organ delineation. Similarly, other artifacts due to motion and metal may have also resulted in contouring errors. As a single, trained reviewer performed the organ delineations with a high degree of consistency, any major errors were likely mitigated. Another limitation is the retrospective nature of the study and the absence of detailed toxicity outcomes to analyze in the context of our organ displacement and dosimetric data. Future prospective evaluation with long term follow up of toxicities as well as assessment of intrafractional volumetric consequences would certainly be needed to validate our hypothesis-generating observation of minimal clinical impact from strict bladder/rectal filling protocols. In terms of measuring organ displacement, an indirect measurement of center of mass changes to contoured volumes had to be employed due to limitations in geometric data generated on the treatment planning system.

Lastly, even with consistent organ delineation by a single reviewer and a large number of $\mathrm{CT}$ images analyzed, the post hoc nature of using clinical CBCTs invariably represents an additional limitation. Namely, the field of view (FOV) of a small number of the CBCTs were optimized for visualization of fiducial markers and pelvic bony landmarks, while minimizing superior and inferior borders to limit image acquisition time and unwarranted radiation exposure. Inevitably, the superior borders of bladders in 69 of 425 CBCTs (16.2\%) throughout all fractions and 10 of 85 fraction \#5 CBCTs (11.8\%) were out of FOV to be precisely contoured. This was due to the FOV not extending superiorly to account for overfilled bladders at the time of image acquisition (average volumes of bladder out of CBCT FOV: $414.5 \pm 116.8 \mathrm{~mL}$; average volumes of bladder within CBCT FOV: $195.8 \pm 105.1 \mathrm{~mL}$ ). The degree of bladder overfilling in these $\mathrm{CBCTs}$ were remarkable enough to limit any major changes to the overall pattern of bladder volume decline, $\mathrm{COM}$, or dosimetry to warrant exclusion of patients from analyses.

\section{Conclusions}

Despite strict bladder filling instructions and a considerably truncated number of treatment days, a highly variable and decreasing trend in interfractional bladder and rectal volumes were observed in this SBRT cohort during the course of therapy. Such patterns in volumetric variations translated to center of mass displacement of the bladder trigone anteriorly and were associated with changes to OAR dosimetry. While statistically significant, the relatively small changes in OAR dose and displacement did not correlate with any detrimental clinical outcomes on our preliminary analyses. This study suggests that future prospective evaluation of the optimal bladder/rectal filling during prostate SBRT is necessary and perhaps less strict bladder filling and rectal emptying protocols may be required for treatment.

\section{Acknowledgements \\ Not applicable.}

\section{Authors' contributions}

$D B, D G, L J, L H, P P, X P$, and $M Z$ made substantial contributions to the conception of the study, data acquisition, statistical interpretation, and drafting of the article. SB and ZZ made substantial contribution to statistical interpretation. All authors made contribution towards drafting of the manuscript, and read and approved the final manuscript prior to submission. All authors read and approved the final manuscript.

\section{Funding}

There are no sources of research funding to disclose.

\section{Availability of data and materials}

The datasets used and/or analysed during the current study are available from the corresponding author on reasonable request.

\section{Ethics approval and consent to participate}

The Institutional Research Board at Memorial Sloan Kettering Cancer Center approved this study.

\section{Consent for publication \\ Not applicable.}

\section{Competing interests}

M.H. receives research funding from Varian Medical Systems. M.Z. is a consultant for Boston Scientific and Editor in-Chief of Brachytherapy. All other authors declare that they have no competing interests.

\section{Author details}

${ }^{1}$ Department of Radiation Oncology, NYU Langone Health, 160 East 34th St, New York, NY, USA. ${ }^{2}$ Department of Radiation Oncology, Memorial Sloan Kettering Cancer Center, 1275 York Ave, New York, NY 10065, USA. ${ }^{3}$ Department of Medical Physics, Memorial Sloan Kettering Cancer Center, 1275 York Ave, New York, NY, USA. ${ }^{4}$ Department of Epidemiology and Biostatistics, Memorial Sloan Kettering Cancer Center, 1275 York Ave, New York, NY, USA.

Received: 22 April 2020 Accepted: 6 October 2020

Published online: 16 October 2020

\section{References}

1. Katz A, Kang J. Stereotactic body radiotherapy with or without external beam radiation as treatment for organ confined high-risk prostate carcinoma: a six year study. Radiat Oncol (Lond). 2014;9:1.

2. Wu QJ, LiT, Yuan L, Yin FF, Lee WR. Single institution's dosimetry and IGRT analysis of prostate SBRT. Radiat Oncol (Lond). 2013;8:215.

3. Bolzicco G, Favretto MS, Satariano N, Scremin E, Tambone C, Tasca A. A single-center study of 100 consecutive patients with localized prostate cancer treated with stereotactic body radiotherapy. BMC Urol. 2013;13:49.

4. King CR, Freeman D, Kaplan I, Fuller D, Bolzicco G, Collins S, et al. Stereotactic body radiotherapy for localized prostate cancer: pooled analysis from a multi-institutional consortium of prospective phase II trials. Radiother Oncol. 2013;109(2):217-21. 
5. Chen LN, Suy S, Uhm S, Oermann EK, Ju AW, Chen V, et al. Stereotactic body radiation therapy (SBRT) for clinically localized prostate cancer: the Georgetown University experience. Radiat Oncol (Lond). 2013;8:58.

6. Zelefsky MJ, Kollmeier M, McBride S, Varghese M, Mychalczak B, Gewanter $R$, et al. Five-year outcomes of a phase 1 dose-escalation study using stereotactic body radiosurgery for patients with low-risk and intermediaterisk prostate cancer. Int J Radiat Oncol Biol Phys. 2019;104(1):42-9.

7. Widmark A, Gunnlaugsson A, Beckman L, Thellenberg-Karlsson C, Hoyer M, Lagerlund M, et al. Ultra-hypofractionated versus conventionally fractionated radiotherapy for prostate cancer: 5-year outcomes of the HYPO-RT-PC randomised, non-inferiority, phase 3 trial. Lancet. 2019;394(10196):385-95.

8. Jackson WC, Silva J, Hartman HE, Dess RT, Kishan AU, Beeler WH, et al. Stereotactic body radiation therapy for localized prostate cancer: a systematic review and meta-analysis of over 6000 patients treated on prospective studies. Int J Radiat Oncol Biol Phys. 2019;104(4):778-89.

9. Meier RM, Bloch DA, Cotrutz C, Beckman AC, Henning GT, Woodhouse SA, et al. Multicenter trial of stereotactic body radiation therapy for low- and intermediate-risk prostate cancer: survival and toxicity endpoints. Int J Radiat Oncol Biol Phys. 2018;102(2):296-303.

10. Alongi F, Mazzola R, Fiorentino A, Corradini S, Aiello D, Figlia V, et al. Phase II study of accelerated Linac-based SBRT in five consecutive fractions for localized prostate cancer. Strahlenther Onkol Organ Deutschen Rontgengesellschaft . 2019;195(2):113-20.

11. Alayed Y, Cheung P, Chu W, Chung H, Davidson M, Ravi A, et al. Two StereoTactic ablative radiotherapy treatments for localized prostate cancer (2STAR): results from a prospective clinical trial. Radiother Oncol. 2019;135:86-90.

12. Cho LC, Timmerman R, Kavanagh B. Hypofractionated external-beam radiotherapy for prostate cancer. Prostate Cancer. 2013;2013:103547.

13. Loblaw A, Cheung P, D'Alimonte L, Deabreu A, Mamedov A, Zhang L, et al. Prostate stereotactic ablative body radiotherapy using a standard linear accelerator: toxicity, biochemical, and pathological outcomes. Radiother Oncol. 2013;107(2):153-8.

14. Shrout PE, Fleiss JL. Intraclass correlations: uses in assessing rater reliability. Psychol Bull. 1979;86(2):420-8.

15. Lebesque JV, Bruce AM, Kroes AP, Touw A, Shouman RT, van Herk M. Variation in volumes, dose-volume histograms, and estimated normal tissue complication probabilities of rectum and bladder during conformal radiotherapy of T3 prostate cancer. Int J Radiat Oncol Biol Phys. 1995;33(5):1109-19.

16. Zellars RC, Roberson PL, Strawderman M, Zhang D, Sandler HM, Ten Haken RK, et al. Prostate position late in the course of external beam therapy: patterns and predictors. Int J Radiat Oncol Biol Phys. 2000;47(3):655-60.

17. Mullaney LM, O'Shea E, Dunne MT, Finn MA, Thirion PG, Cleary LA, et al. A randomized trial comparing bladder volume consistency during fractionated prostate radiation therapy. Pract Radiat Oncol. 2014;4(5):e203-12.

18. Zelefsky MJ, Crean D, Mageras GS, Lyass O, Happersett L, Ling CC, et al. Quantification and predictors of prostate position variability in 50 patients evaluated with multiple CT scans during conformal radiotherapy. Radiother Oncol. 1999;50(2):225-34.

19. Pinkawa M, Asadpour B, Gagel B, Piroth MD, Holy R, Eble MJ. Prostate position variability and dose-volume histograms in radiotherapy for prostate cancer with full and empty bladder. Int J Radiat Oncol Biol Phys. 2006;64(3):856-61.

20. De Meerleer GO, Villeirs GM, Vakaet L, Delrue LJ, De Neve WJ. The incidence of inclusion of the sigmoid colon and small bowel in the planning target volume in radiotherapy for prostate cancer. Strahlenther Onkol Organ Deutschen Rontgengesellschaft. 2004;180(9):573-81.

21. Nakamura N, Shikama N, Takahashi O, Ito M, Hashimoto M, Uematsu M, et al. Variability in bladder volumes of full bladders in definitive radiotherapy for cases of localized prostate cancer. Strahlenther Onkol Organ Deutschen Rontgengesellschaft. 2010;186(11):637-42.

22. Stam MR, van Lin EN, van der Vight LP, Kaanders JH, Visser AG. Bladder filling variation during radiation treatment of prostate cancer: can the use of a bladder ultrasound scanner and biofeedback optimize bladder filling? Int J Radiat Oncol Biol Phys. 2006;65(2):371-7.

23. O'Doherty UM, McNair HA, Norman AR, Miles E, Hooper S, Davies M, et al. Variability of bladder filling in patients receiving radical radiotherapy to the prostate. Radiother Oncol. 2006;79(3):335-40.

24. Repka MC, Kole TP, Lee J, Wu B, Lei S, Yung T, et al. Predictors of acute urinary symptom flare following stereotactic body radiation therapy (SBRT) in the definitive treatment of localized prostate cancer. Acta Oncol. 2017:56(8):1136-8.

25. Ghadjar P, Zelefsky MJ, Spratt DE, Munck af Rosenschold P, Oh JH, Hunt M, et al. Impact of dose to the bladder trigone on long-term urinary function after high-dose intensity modulated radiation therapy for localized prostate cancer. Int J Radiat Oncol Biol Phys. 2014;88(2):339-44.

26. Hathout L, Folkert MR, Kollmeier MA, Yamada Y, Cohen GN, Zelefsky MJ. Dose to the bladder neck is the most important predictor for acute and late toxicity after low-dose-rate prostate brachytherapy: implications for establishing new dose constraints for treatment planning. Int J Radiat Oncol Biol Phys. 2014;90(2):312-9.

27. Pos FJ, Koedooder K, Hulshof MC, van Tienhoven G, Gonzalez GD. Influence of bladder and rectal volume on spatial variability of a bladder tumor during radical radiotherapy. Int J Radiat Oncol Biol Phys. 2003;55(3):835-41.

28. Pinkawa M, Asadpour B, Siluschek J, Gagel B, Piroth MD, Demirel C, et al. Bladder extension variability during pelvic external beam radiotherapy with a full or empty bladder. Radiother Oncol. 2007;83(2):163-7.

29. Antolak JA, Rosen II, Childress CH, Zagars GK, Pollack A. Prostate target volume variations during a course of radiotherapy. Int J Radiat Oncol Biol Phys. 1998;42(3):661-72.

30. D'Agostino G, Franzese C, De Rose F, Franceschini D, Comito T, Villa E, et al. High-quality linac-based stereotactic body radiation therapy with flattening filter free beams and volumetric modulated arc therapy for low-intermediate risk prostate cancer. A mono-institutional experience with 90 patients. Clin Oncol (R Coll Radiol). 2016;28(12):e173-8.

31. Wahl M, Descovich M, Shugard E, Pinnaduwage D, Sudhyadhom A, Chang $A$, et al. Interfraction anatomical variability can lead to significantly increased rectal dose for patients undergoing stereotactic body radiotherapy for prostate cancer. Technol Cancer Res Treat. 2017;16(2):178-87.

32. Swanson GP, Stathakis S. Rectal dose constraints for intensity modulated radiation therapy of the prostate. Am J Clin Oncol. 2011;34(2):188-95.

33. Wallner K, Roy J, Harrison L. Dosimetry guidelines to minimize urethral and rectal morbidity following transperineal I-125 prostate brachytherapy. Int J Radiat Oncol Biol Phys. 1995;32(2):465-71.

34. Kim DW, Cho LC, Straka C, Christie A, Lotan Y, Pistenmaa D, et al. Predictors of rectal tolerance observed in a dose-escalated phase 1-2 trial of stereotactic body radiation therapy for prostate cancer. Int J Radiat Oncol Biol Phys. 2014;89(3):509-17.

35. Madsen BL, Hsi RA, Pham HT, Fowler JF, Esagui L, Corman J. Stereotactic hypofractionated accurate radiotherapy of the prostate (SHARP), 33.5 Gy in five fractions for localized disease: first clinical trial results. Int J Radiat Oncol Biol Phys. 2007;67(4):1099-105.

36. McBride SM, Wong DS, Dombrowski JJ, Harkins B, Tapella P, Hanscom HN, et al. Hypofractionated stereotactic body radiotherapy in low-risk prostate adenocarcinoma: preliminary results of a multi-institutional phase 1 feasibility trial. Cancer. 2012;118(15):3681-90.

37. Radiation Therapy in Treating Patients With Prostate Cancer (NCT01434290) (Internet). 2016. https://clinicaltrials.gov/show/NCT01 434290.

38. Radiation Hypofractionation Via Extended Versus Accelerated Therapy (HEAT) For Prostate Cancer (NCT01794403) (Internet). 2016. https://clini caltrials.gov/ct2/show/NCT01794403.

\section{Publisher's Note}

Springer Nature remains neutral with regard to jurisdictional claims in published maps and institutional affiliations. 